\title{
Critical Analysis and Communication: an alternative to the final year project
}

Authors: Jill Barber, Constantinos Demonacos, Leon Aarons, Douglas Steinke, Mary Tully. Manchester Pharmacy School, The University of Manchester

Background: The MPharm programme requires that students receive a training in research methods. This is normally achieved through the Research Project in which students conduct, evaluate and report a piece of original research. The Research Project is hugely valuable for some students, but others expecting to practise as health care professionals find the development of specialised practical or computational skills a distraction. They do, however, need other research skills. Critical Analysis and Communication focusses on those core research methods required by a healthcare professional serving the public.

Method: In developing this 30-credit module our objectives were:

- To train students to evaluate research data critically, whether presented in peer-reviewed journals, or in the news media.

- To train students to communicate their analyses clearly and with consideration of the patient's needs. To enable students to develop confidence in their abilities regarding clinical decisions in an ethical framework.

- To create a module that rewards hard work by adapting a meaningful marking scheme.

In semester 1 the class was introduced to the possibility of bias in different settings e.g clinical trials, biological research, statistics, through lectures by experts. Oral and written analysis and communication were assessed. In semester 2, students prepared a protocol for a hypothetical research project, addressing ethical and statistical concerns at a level of detail that is not always possible in a research project that can occupy only two semesters.

Results: Students not only performed well in the module (final marks mean 69\%, SD $6.7 \%$ ), but exceeded expectations in other fourth year modules (6 of 23 students rising one degree class relative to the third year) and they developed skills very relevant to their future careers (14/20 students strongly agree, 6/20 agree).

Conclusion: Critical Analysis and Communication is not intended to replace the Research Project, but is an excellent alternative for many students. 